\title{
An Energy and Traffic Aware Routing Approach as an Extension of AODV
}

\author{
Kapil Kumar \\ Department of Computer \\ Science \& Engineering, \\ Madan Mohan Malaviya \\ Engineering College, \\ Gorakhpur-273010, U.P., INDIA
}

\author{
Shiva Prakash \\ Department of Computer \\ Science \& Engineering, \\ Madan Mohan Malaviya \\ Engineering College, \\ Gorakhpur-273010, U.P., INDIA
}

\author{
Sumit Kumar Singh \\ Department of Computer \\ Science \& Engineering, \\ Madan Mohan Malaviya \\ Engineering College, \\ Gorakhpur-273010, U.P., INDIA
}

\begin{abstract}
The importance of Ad-hoc network is due to its nature of infrastructure-less and decentralization. In Ad-hoc network some nodes can become a critical spot in the network as they forward the packets to most of their neighbors. These critical nodes can deplete their battery power earlier, because of excessive load and processing for data forwarding. These unbalanced loads turn to nodes failure, network partition and reduce the route lifetime and route reliability. Energy consumption issue is a vital research topic in wireless ad hoc networks, because wireless nodes in such networks operate on limited battery power. This model paper describes An Energy and Traffic Aware Routing approach as an extension of AODV algorithm for Ad hoc network, utilizing the high energy path based on Route Selection Function. Our proposed algorithm adapts existing AODV routing protocol to improve performance in terms of traffic load on node, energy conservation and other performance metrics. The purpose of Route Selection Function is to improve lifespan of Ad hoc network and corresponding effect on overall network performance.
\end{abstract}

\section{Keywords}

AODV, Traffic load, Remaining Energy.

\section{INTRODUCTION}

Wireless Mobile Ad hoc network [1] enable nodes to setup a network quickly, provides advantage in deployment, cost, size and distributed intelligence over wired networks. It is a challenging task to provide the same type of services and same quality in wireless mobile environments as in wired environment. Most of earlier work on routing in ad hoc networks deal with the problem of finding and maintaining correct routes to the destination due to mobility and changing topology. Majority of routing protocols in mobile ad hoc networks use min-hop routing where the number of hops is the path length. A network's capability to provide a particular quality of service between a set of endpoints depends upon the inherent performance properties (e.g. delay, throughput, loss rate, error rate) of the links and nodes, the traffic load within the network and the control algorithms operating at different layers of the network. Ad hoc routing protocols can broadly be classified as Table driven and on demand routing protocols. Table driven routing protocols attempt to maintain consistent, up to date routing information for the nodes in the network. In these protocols each node maintain one or more tables to store routing information, whenever any changes in network topology then they respond by propagating updates messages throughout the network in order to maintain consistent network view. On the other hand On demand routing creates routes only when required by the source node. When a node requires a route to a destination, it initiates a route discovery process within the network. The process is completed when a route is found or all possible routes have been examined. Once the route has been established, some form of route maintenance procedure maintains it until the route is no longer desired. Well-known routing protocols studied under on demand category are AODV [2] and DSR [3] (Dynamic Source Routing) while DSDV (Destination Sequenced Distance Vector routing) represents table driven category. AODV is an improvement on DSDV because it typically minimizes the number of required broadcasts by creating routes on an on-demand basis, as opposed to maintaining a complete list of routes as in DSDV routing protocol. DSR has higher connection setup delay and its performance degrades rapidly with increasing mobility as compared to AODV. AODV uses destination sequence number to find the latest route to destination requiring less setup delay also fair performance with mobility concern. Traditional routing protocols for ad hoc network select the routes under the metric of the minimum hop count. Such min-hop routing protocols can use energy unevenly among the nodes and thus it can cause some nodes to spend their whole energy earlier and make a network partition as a degradation in network performance. End to end delay and throughput are commonly used performance metrics in wired and wireless networks. Since network topology change dynamically, bandwidth and battery power are additional important factors to be considered in wireless ad hoc networks. If energy consumption is to be considered, then best suited protocols are on-demand protocols where control information transfer is limited and is not as frequently updated as in table driven protocols or proactive protocols. On demand protocols flooding the route request packets throughout the network does the route discovery. Our main aim is to make the routing protocol more energy efficient and ultimately to perform load balancing of the node in the ad hoc network.

\subsection{AODV}

Ad hoc On Demand Distance Vector (AODV)[2], routing algorithm comes under the category of On- demand routing protocol means that it creates routes between nodes only as needed by source nodes, designed for ad hoc mobile networks. It 
is capable of both unicast and multicast routing. It maintains routes as long as they are required by the sources. It also forms trees which join multicast group members. The trees are collected of the group members and the nodes needed to connect the members. AODV has sequence numbers, which ensure the freshness of routes. It provides loop-free routing using broadcast ID. AODV make routes, using a route request / reply query cycle. When a originator node wants a route to a destination node for which it does not have a route, it broadcasts a route request (RREQ) packet throughout the network. Nodes receiving RREQ packet update their information for the originator node and set up backwards pointers to the originator node in the route tables. In addition to the originator node's current sequence number, IP address and broadcast ID, the RREQ contains the most recent sequence number for the destination of which the originator node is aware. A node receiving the RREQ may send a route reply (RREP) if it is either the destination node or if it has a route to the destination node with corresponding sequence number greater than or equal to that enclosed in the RREQ. If this case exist, it unicast a RREP back to the originator node. Otherwise, it rebroadcasts the RREQ. Nodes keep track of the RREQ's broadcast ID and source IP address. If they get a RREQ which they have already processed, they discard the RREQ and no need to forward it. As the RREP propagates back to the originator node, nodes set up forward pointers to the destination node. Once the originator node receives the RREP, than the path is established between originator node to destination node and further originator can utilize this path for data transmission. Later if the originator node receives RREP with greater sequence number or contains the same sequence number with a smaller hop count, it may update its routing table entry for that destination node and use the fresh route. As long as the route remains active, it will continue to be maintained. A route is considered active as long as there are data packets periodically travelling from the originator node to the destination node along that path. Once the originator node stops sending data packets, the links will time out and eventually be deleted from the intermediate node routing tables. If a link break occurs while the route is active, the node upstream of the break propagates a route error (RERR) message to the originator node to inform it of the now unreachable destination(s). After receiving the RERR, if the originator node still desires the route, it can reinitiate route discovery.

Rest of the paper organised as follows. Section II gives the Literature Survey. Section III introduces proposed work.

In section IV discussion and analysis of ETR-AODV is given. Section V shows the conclusion and future work..

\section{LITERATURE REVIEW}

In Ad hoc network the nodes can make a network without any central access point, So there need a different type of routing protocols apart from the conventional routing protocols as in wired networks. Many routing protocols exist in ad hoc Network, these protocols are based on min-hop count but it may not be the best routing criteria with respect to the energy and traffic load of a node because wireless nodes in Ad hoc networks operate on limited battery power.

AODV [2] and DSR [3] are min hop routing protocols. Tai Hieng [4] proposed an algorithm which is an improvement of energy efficient routing by selecting high energy paths, taking account of energy conservation and other performance metrics. Energy metric is used as route selection method to improve lifespan of ad hoc network. Route selection is one of the main domain where many researchers are trying to improve performance of Ad hoc network. In [5] the authors proposed a new metric which consider as the stability of the paths by taking three parameters viz 'affinity', 'available bandwidth' and 'battery level' in routing decisions. It also maintains multipath to achieve load sharing. The routing table in AODV, maintains only one route to specified node, therefore the source node needs to reinitiate route discovery process as a route fails. Luo Chao \& Liping'an [6] presented an improved method, each source will maintain backup routes, when the primary route fails the source node will use the backup route to send packets, which improves the packet delivery ratio and end to end delay. According to different route selection mechanism, energy based routing algorithm can be categories as, minimum total energy consumption which select the route with minimum total energy consumption between source to destination, In which some nodes taking part in transmission may run out of power and leads the partition of networks. Another is maximizing network lifetime algorithm, it establish the route by avoiding the lower energy node so that it can balance the energy consumption of nodes and enhance the lifetime of networks. The final is mixed optimizing routing algorithm which mix the above two categories.

Yonghui Chen [7] proposed energy saving routing protocol named EEAODV based on AODV. In routing discovery process source node consider the remaining energy of intermediate nodes and also consider the influence of these nodes, which may cause route changed. In [8] the authors have given an effective scheme to balance the node in network. This new scheme can be applied in most On-demand routing protocols, It is implement in the process of route request, when RREQs packets are flooded to establish routes, only the qualified nodes, which have a potential to serve as intermediate forwarding nodes will respond to these packets. So that the established path will not be very congested and traffic load will be distributed evenly in the network it also considers a threshold value, which is used to judge whether intermediate node is overloaded, is variable and changing along the nodes interface queue occupancy around the backward path. Most of ad-hoc routing protocols do not consider contention time, occurs in medium reservation procedure. Long contention times can be more critical than hop counts in determining the end to end delay. Some mobile nodes may lead to long queuing delays, low packet delivery ratio and inefficient power consumption. Bong Chan Kim[9] proposed routing protocol with minimum contention time and load balancing (MCL). This protocol has two main functions, MCL selects a route with minimum contention among many possible routes in the route selection procedure and secondly intermediate nodes do not reply to RREQs in the route discovery procedure. MCL outperforms in term of packet delivery ratio, average end to end delay, and normalized routing overhead. Unbalanced traffic may lead to more delay, packet dropping and decreasing packet delivery ratio and unbalanced energy consumption leads to node failure, network partitioning, and decrease route reliability. In [10] authors suggested an approach to improve the performance of routing protocols with respect to, traffic balance energy consumption balance, end to end delay and route reliability. In [12] the author presented a protocol with an improved route discovery mechanism that avoids the congestion by selecting a route on the basis of traffic load and resets path as topology changes, new efficient paths are discovered from time to time 
during transmission. It is an efficient technique for transmission that requires a link for longer period of time. Author in [13] suggested an energy efficient Ad-hoc On-demand routing(EEAODR) algorithm that balances energy load so that a minimum energy level is maintained among nodes and the network lifetime is increased by distributing energy consumption in the network.

As per above literature survey, we found that no any protocol perform efficiently in each and every situation. Now here we propose a solution which is considering the power level of node as well as congestion on node. So, our protocol performs better for finding out the routing path with better energy and low congestion path. Our protocol tries to establish balance of energy among the nodes as well as traffic load in the path. We will discuss the propose routing mechanism in section III.

\section{PROPOSED WORK}

An Energy and Traffic Aware Routing approach as an extension of AODV. The study of literature, inspire us to develop a route selection mechanism which optimized energy and traffic of the node. We consider the following modification for designing our Algorithm.

\subsection{Control message:}

A reserved congestion field and an energy field of node is inserted in the RREQ or RREP. Whenever a node receives RREQ, it appends current energy value in the reserved field and congestion field.

\subsection{Route table of node:}

Two new fields are added in the routing table entry for each node which stores remaining energy and congestion factor. The modified RREQ or RREP message value can be extracted from the route table entry.

\subsection{Route selection process:}

When a destination receives route request it will extract the information of CF and PF from the RREQ. The destination will now calculate Length $\left(\mathrm{L}_{\mathrm{i}}\right)$ and congestion factor of the path of each RREQ received by it on the basis of equation 1and 2 respectively. Now destination will finally select the most energy efficient and less congestion route by the route selection function given in equation 5 .

For a particular source to destination node there exist i routes. The Route Selection Function has three parameters: Length, Traffic Load, and Remaining Energy.

\subsubsection{Length $\left(L_{i}\right)$}

$\mathrm{L}_{\mathrm{i}}$ in equation 1 indicate the selection of route $\mathrm{i}$ with respect to the length of route:

$L_{i}=\frac{\text { Actual_Length_Route } i}{\text { Max_Length }}$

Actual_Length_Route of route (i) is the actual length of route i (i.e. no. of hops in route i) and Max_Length is the maximum length that a route can take in AODV routing protocol.

\subsubsection{Congestion Factor $(C F)$}

To calculate Congestion Factor we define several terms:

$C F_{j}$ : Congestion Factor of node $\mathrm{j}$

$V Q_{j}$ : Vacant interface queue size of node $\mathrm{j}$
$T Q_{j}$ : Total interface queue size of node $\mathrm{j}$

$D Q_{j}$ : No. of data packets in interface queue of node $\mathrm{j}$

The congestion factor is calculated by the following equation,

$C F_{j}=V Q_{j} / T Q_{j}$

Where $V Q_{j}=T Q_{j}-D Q_{j}$

Congestion Factor in equation (2), is the percentage of the network interface queue that is vacant. The default total size of network interface queue is 25 .

$C F_{\text {sdi }}$ value of all nodes along a valid path are multiplied to obtain the congestion factor value for this path.

$$
C F_{s d i}=\prod_{j \in N_{s d i}} C F_{j}
$$

The $C F_{\text {sdi }}$ considers available capacity of the network interface queue as an indication of traffic condition of the route.

Higher value $C F_{s d i}$ indicates that the path has lower traffic load to handle. Such paths should be chosen as it avoids the use of congested paths which leads to higher packet loss ratio, and longer delay.

\subsubsection{Power Factor (PF)}

As we know that mobile Ad hoc network consists of battery constrained mobile nodes. Hence the power factor is an important metric to be considered for route selection. Thus, a route with maximum residual battery power in its node can be an ideal path with respect to energy level. Moreover, despite the fact that sometime a path may have a high residual energy level, it may have some nodes with very low energy levels too. So, there is high probability of failure of these low residual energy nodes which make them undesirable.

In equation (4), the PF indicates the selection of route $i$ with respect to residual energy level.

$P F_{s d i}$ : Power factor for a particular source and destination pair of path $\mathrm{i}$

$E A_{s d i}$ : total of available energy in the nodes in path $\mathrm{i}$

$N_{s d i}:$ Number of nodes from source to destination in path $\mathrm{i}$

$P F_{\text {sdi }}=\frac{E A_{\text {sdi }}}{N_{\text {sdi }} \text { Initial Energy }}$

\subsubsection{Route Selection Function}

The destination node will wait for $\Delta \mathrm{t}$ time for the arrivals of RREQs, then computes the route selection function for all available routes to the destination. The route with maximum Route Selection Function value will be selected as the candidate route. The Route Selection Function is given by following equation:

Route Selection Function $(\mathrm{i})=\frac{P F_{s d i}+C F_{s d i}}{L_{i}}$

From the above equation (5), we can achieve higher performance with regards to end to end delay, traffic load and energy consumption.

\section{PSEUDO-CODE FOR PROPOSED ALGORITHM:}

A node wants to transmit data packet:

If 
(path to destination is found in route table and it has not expired)

Go to L4:

Else

// Find the path

L1: Broadcast RREQ to neighbor nodes whose available energy $\geq 30 \%$ with modifications valid RREQ are received by nodes.

If $R R E Q$ receiving node is the destination.

If RREP has been sent

It is duplicate RREQ, reject it.

Else

If it is the first RREQ from the source

Endif Setup the timer for $\Delta t$ duration

Do the corresponding entry in seen table. Endif

Else node is intermediate one

Do the corresponding entry in seen table.

Jump to L1

Endif

At destination node:

If time interval $\Delta t$ passes

Calculate best path on the basis of Route Selection Function.

Send RREP
At source node:

If Source receive RREPs within timeout interval Source updates its routing table with selected path

L4: Source transmits data through the selected path

Else

Send RREQ again or logout. Endif

We present discussion and analysis of ETR-AODV in the next section.

\section{DISCUSSION AND ANALYSIS}

The ETR-AODV Routing algorithm is based on AODV. The route discovery procedure in AODV protocol is modified, to select the high energy path on the basis of route selection function by the destination node. The route maintenance procedure is essentially the same as in AODV protocol.

Generally in On-demand routing algorithm [1][2], when a source needs to know the route to a destination it broadcasts a RREQ packet. The neighboring nodes receiving the RREQ, relay this immediately to its neighbors. But the idea of ETRAODV algorithm is as follows: Before relaying the packet, each intermediate node calculates congestion factor(CF) and available energy(EA) value and add it to respective reserved field in RREQ. Fig.1 is explaining the whole process of RREQ.

L2: Wait for new valid RREQ until $\Delta$ t times out.

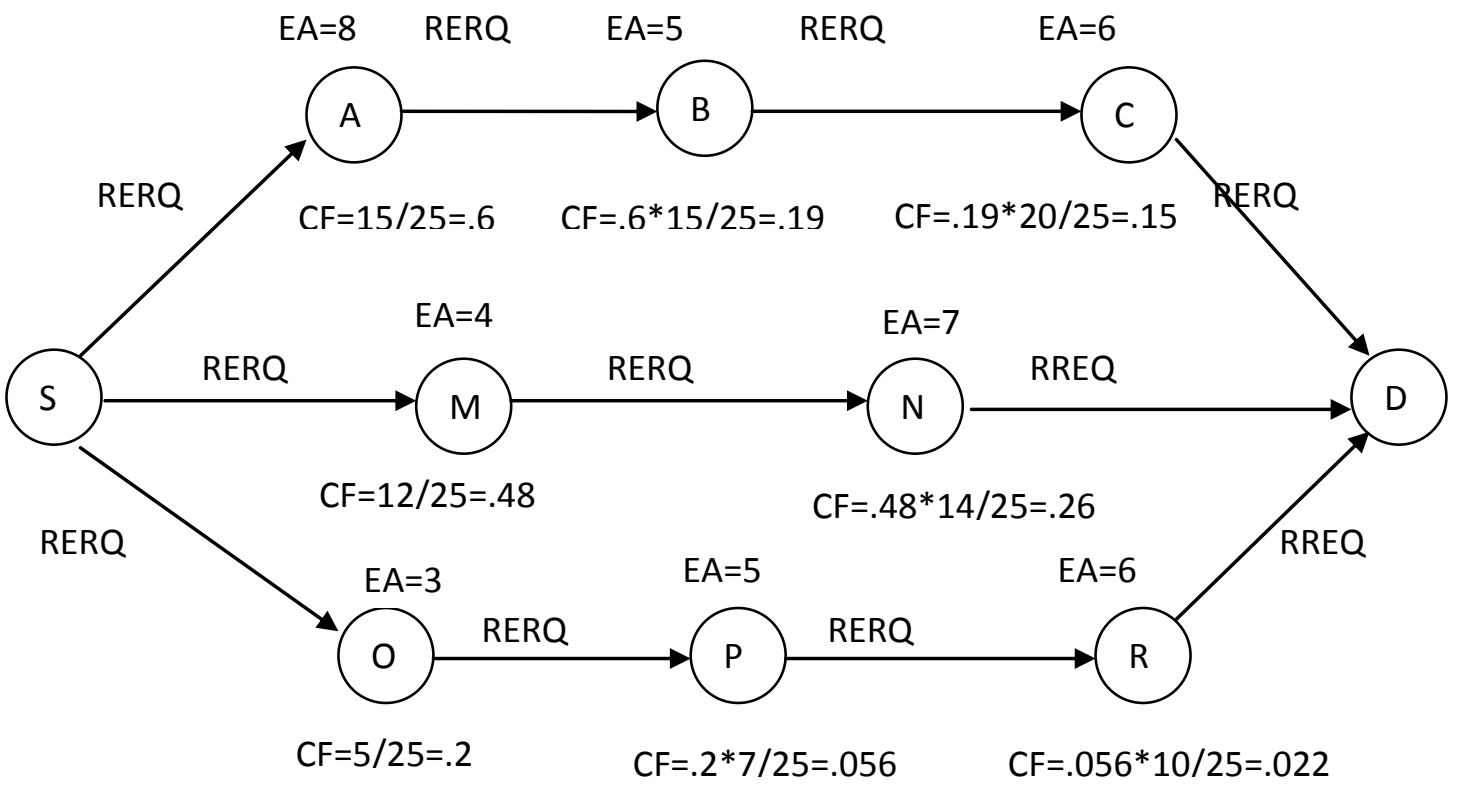

Fig. 1

In Fig. 2.The RREP is unicast to the source. On the basis of Power factor and congestion factor values obtained from RREQ, the destination determine the route selection function of each path. The route with highest function value will be considered as candidate path, through which the data transmission will be done. Thus route S-M-N-D will be selected as candidate path, as it has the higher value of route selection function i.e. $\mathrm{RS}_{2}=1.08$. 


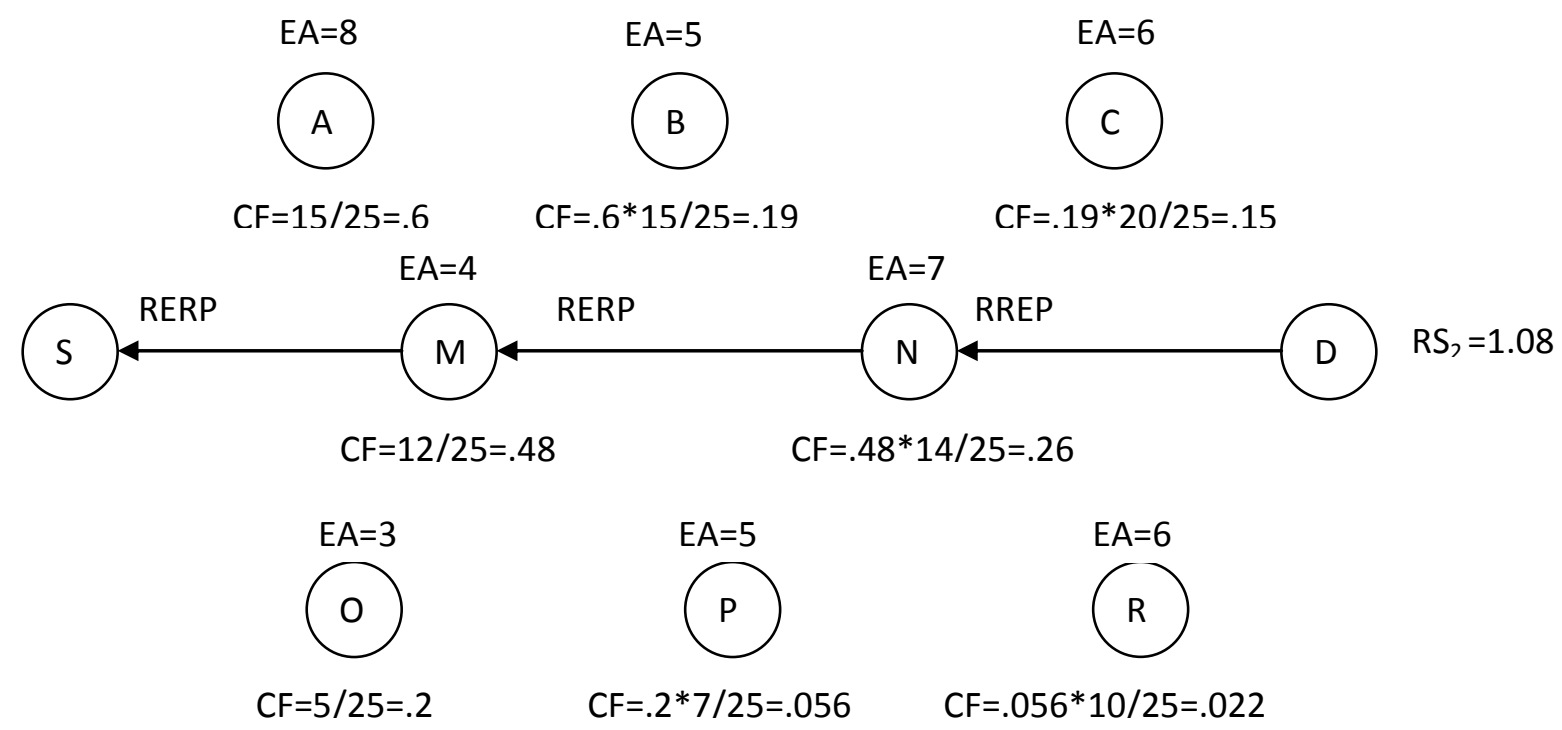

Fig.2

\section{CONCLUSION AND FUTURE WORK}

In this model paper, we proposed a modified algorithm based on AODV. In this algorithm we have modified the route selection procedure, the destination node unicast the route reply to the path, having less congestion and maximum energy. ETR-AODV protocol with enhanced route discovery mechanism which takes into account the energy and congestion factor to select the most efficient route. Thus, rather than using the traditional metrics such as delay or hop count, we believe the node's energy and traffic load to be taken in to account as routing metric in a integrated way which can give the better performance in MANET. ETR-AODV attempts to find more suitable routes to the destination by choosing the paths with a maximum residual battery and minimum traffic load. This protocol may extend network lifetime, route availability and increase the packet delivery ratio. Simulation of ETR-AODV is our future work.

\section{REFERENCES}

[1] I. Chakeres, J. Macker, T.Clausen "Mobile Ad hoc Network Architecture",draft-ietf-autoconf-manetarch-07.

[2] C. E. Perkins, S R Das and E Royer, “Ad-hoc On Demand Distance Vector(AODV) Rousting”, RFC 3561.

[3] D. B. Johnson, D.A. Maltz, and Y. Hu, The Dynamic Source Routing protocol (DSR) for Mobile Ad hoc networks for IPv4, IETF RFC 4728, Feb. 2007.

[4] Tai Hieng Tie et al., "Maximum Energy Level ad hoc distance vector scheme for energy efficient ad hoc networks routing", proceedings of the 2009 IEEE $9^{\text {th }}$ Malaysia International Conference on Communications 1517 December 2009, Kuala Lumpur Malaysia.
[5] N Thanthry, S R Kaki and R Pendse, "EM- AODV: Metric Based enhancement to AODV routing protocol", IEEE 2006.

[6] Luo Chao, Li Ping'an, "An Efficient Routing approach as an extension of the AODV Protocol", IEEE 2010.

[7] Yonghui Chen and Chunfeng Zhang, "Energy efficient routing protocol for ad hoc networks" IEEE, International conference on computer design and applications (ICCDA 2010), Volume 5, 2010.

[8] YuHua Yuan, HuiMin Chen and Min Jia, "An adaptive load balancing approach for ad hoc networks", IEEE 2005.

[9] Bong Chan Kim et al., "An ad hoc routing protocol with minimum contention time and load balancing ", GLOBECOM, IEEE 2003.

[10] Vahid Nazari Talooki, Jonathan Rodriguez, Rasool Sadeghi, "A Load balanced aware routing protocol for wireless ad hoc networks", IEEE 2009.

[11] Vahid Nazari Talooki, Jonathan Rodriguez, Hugo Marques, "An energy efficient flat routing protocol for wireless ad hoc networks", IEEE 2010.

[12] Iftikhar Ahmad and Mata ur Rehman, "Efficient AODV routing based on traffic load and mobility of node in MANET", IEEE $20106^{\text {th }}$ International Conference on emerging technologies (ICET).

[13] Sanjay Kumar Dhurandher, Sudip Misra et al., “ An Energy-Efficient On Demand Routing Algorithm For Mobile Ad Hoc Networks", IEEE 2008. 